workers, was implemented under the USAID supported Samastha project (2006-2011).

Methods A rapid rural mapping covering 7700 villages across eight districts helped map 16043 rural female sex workers clustered in about 1700 villages. Rural female sex workers (RFSW) were defined as women who lived and practiced sex work within the village. Peer educators supervised by outreach link workers identified, educated, directly provided condoms and mobilised RFSW to reach HIV services. STI and counselling and testing services were integrated into Primary Health Centres. An individualised web based management information system tracked outreach and services. Polling booth surveys were used to measure key behavioural outcomes.

Results By the fourth year of implementation, 14697 (91.6\%) of the estimated RFSW were reached. Monthly contact rates were sustained at $85 \%$, with each RFSW directly receiving a mean of 28.3 condoms per month. Condom use rates rapidly increased to $63 \%$ at last sex with any partner and $78 \%$ with clients. $81 \%$ received STI services and more than $67 \%$ had been directly referred and received their HIV status. The proportion reporting with STI symptoms remained stable at around $60 \%$; however, $64 \%$ of these were vaginal discharge. Genital ulcer rates decreased from 5.5 to $0.8 \%$ and other STI conditions from 6.1 to $1.7 \%$. HIV prevalence among RFSW across districts ranged from 2 to $4 \%$. Overall, HIV prevalence among antenatal women declined from 1.9 to $0.5 \%(2006-2009)$ in these districts.

Conclusions Focused and early interventions can reduce HIV transmission in rural areas and could be impacting even at the population level. It would be important to sustain focused prevention interventions in rural India.

\section{P2-S2.12 CONDOM USE WITHIN INTIMATE PARTNERSHIPS OF FEMALE SEX WORKERS IN SOUTHERN INDIA}

\section{doi:10.1136/sextrans-2011-050108.308}

${ }^{1} \mathrm{~K}$ Deering, ${ }^{2} \mathrm{P}$ Bhattacharjee, ${ }^{2} \mathrm{R}$ Washington, ${ }^{3} \mathrm{~J}$ Bradley, ${ }^{4} \mathrm{M} \mathrm{C}$ Boily, ${ }^{5} \mathrm{~S}$ Shaw, ${ }^{1} \mathrm{~K}$ Shannon, ${ }^{2} \mathrm{~B} \mathrm{M}$ Ramesh, ${ }^{2} \mathrm{~S}$ Rajaram, ${ }^{5} \mathrm{~S}$ Moses, ${ }^{3} \mathrm{M}$ Alary. ${ }^{1}$ University of British Columbia, Vancouver, Canada; ${ }^{2}$ Karnataka Health Promotion Trust, Bangalore, India; ${ }^{3}$ Laval University, Canada; ${ }^{4}$ Imperial College, UK; ${ }^{5}$ University of Manitoba, Winnipeg, Canada

Background Although female sex workers (FSWs) report high levels of condom use with commercial sex clients, particularly after targeted HIV preventive interventions have been implemented, condom use is often low with intimate partners. There is limited understanding regarding the factors that influence condom use with FSWs' non-commercial intimate partners, and how programs can be designed to increase condom use. The main objectives of this study were therefore to describe FSWs' self-reported intimate partners, along with interpersonal factors characterising their intimate partnerships, and to examine factors associated with inconsistent condom use within intimate partnerships.

Methods This study used data collected from cross-sectional questionnaires administered to 988 FSWs in four districts in Karnataka state in 2006. We used bivariate and multivariable logistic regression analysis to examine the relationship between inconsistent condom use (ie, "never", "sometimes" or "frequently", compared to "always") with intimate partners of FSWs (including husbands/cohabiting partners and other non-paying partners) and interpersonal factors describing these partnerships. Weighting and survey methods were used to account for the cluster sampling design.

Results Overall, 511 (51.8\%) FSWs reported having husbands/ cohabiting partners and $273(26.0 \%)$ reported having other nonpaying partners. Inconsistent condom use with these partners was high $(77.4 \%$ and $60.4 \%$ respectively). In multivariable analysis, adjusting for social and environmental factors, the odds of inconsistent condom use with husbands/cohabiting partners and other non-paying partners were $12 \%$ (adjusted OR [AOR]: 1.12, 95\% CIs 1.06 to 1.17 ) and 35\% (AOR: $1.35,95 \%$ CI 1.13 to 1.62 ) higher for a one-year increase in the duration of the relationship, respectively. The odds of inconsistent condom use with husbands/cohabiting partners was $50 \%$ lower if these partners knew that the respondent was a sex worker (AOR: $0.50,95 \%$ CI 0.29 to 0.86 ). The odds of inconsistent condom use with other non-paying partners was $68 \%$ lower if the respondent reported ever having stayed or lived with these partners (AOR: $0.32,95 \%$ CI 0.13 to 0.79 ).

Conclusions Improved designs for HIV preventive programs, including partner- or couples-focused programs, should be developed to address issues related to FSWs' intimate partnerships and increase condom use.

\section{P2-S2.13 A PILOT STUDY OF THE EFFECTIVENESS OF A VAGINAL WASHING CESSATION INTERVENTION AMONG KENYAN FEMALE SEX WORKERS}

doi:10.1136/sextrans-2011-050108.309

${ }^{1} \mathrm{~L}$ Masese, ${ }^{1} \mathrm{R}$ S McClelland, ${ }^{2} \mathrm{R}$ Gitau, ${ }^{1} \mathrm{G}$ Wanje, ${ }^{2} \mathrm{~J}$ Shafi, ${ }^{2} \mathrm{~F}$ Kashonga, ${ }^{2} \mathrm{~J}$ NdinyaAchola, ${ }^{1} \mathrm{~B}$ Richardson, ${ }^{3} \mathrm{R}$ Lester, ${ }^{4} \mathrm{~A}$ Kurth. ${ }^{1}$ University of Washington, Seattle, USA; ${ }^{2}$ University of Nairobi, Kenya; ${ }^{3}$ University of British Columbia, Canada; ${ }^{4}$ New York University, USA

Background Intravaginal practices have been associated with HIV-1 acquisition. This may be mediated by mucosal disruption, changes in vaginal flora, or inflammatory responses in the genital tract. Reducing vaginal washing could lower women's risk of HIV-1 acquisition. We conducted a prospective study to test the hypothesis that a theory-based intervention would reduce vaginal washing in a cohort of high-risk Kenyan women. We collected pilot data on changes in biological markers that might help to explain the relationship between vaginal washing and HIV-1.

Methods HIV-1 seronegative women who reported current vaginal washing were recruited from a prospective cohort study of high-risk women in Mombasa, Kenya. A theoretical framework including Information Motivation and Behaviour and Harm Reduction was implemented to encourage participants to reduce or eliminate vaginal washing. At baseline and after 1 month, we evaluated vaginal epithelial lesions by colposcopy, vaginal flora by Nugent's criteria, and vaginal cytokine milieu using ELISA on cervicovaginal lavage specimens.

Results Twenty-three women were enrolled. The most commonly reported vaginal washing substance was soap and water $(\mathrm{N}=14$, $60.9 \%$ ). The median frequency of vaginal washing per week was 7 (IOR 0-14). After one week, 21 (91.3\%) participants reported cessation of vaginal washing. After 1 month, all participants reported cessation of vaginal washing ( $\mathrm{p} \leq 0.001$ for comparison of baseline to follow-up prevalence). The average number of cervicovaginal epithelial lesions by colposcopy decreased after 1 month compared to baseline (Mean [SD] 0.4 [0.6] vs 0.2 [0.5]; coefficient $-0.14 ; 95 \%$ CI -0.29 to $0.01 ; p=0.08)$. Although there was no change in the prevalence of BV (OR 1.00, 95\% CI 0.42 to 2.38; $\mathrm{p}=1.00]$ ), these pilot data suggest that the likelihood of detecting Lactobacillus by culture might increase after cessation of vaginal washing (2 [8.8\%] vs 6 [26.1\%]; OR 3.71, 95\% CI 0.73 to 18.76 , $\mathrm{p}=0.11$ ). Most cytokine levels were reduced after cessation of vaginal washing, but in this small, time-limited sample none of these changes were statistically significant.

Conclusions A theory-based intervention was highly successful in reducing vaginal washing over 1 month. This pilot study suggests the need for future studies with a larger sample size and longer follow-up to determine the effects of vaginal washing cessation on 